\title{
Reseñas en revistas científicas españolas de Ciencias Sociales y Humanidades: análisis de la producción entre 2007 y 2009
}

\author{
Ángela Sorli Rojo*, Gonzalo Mochón Bezares*, Carolina Martín-Carretero*
}

Resumen: Se presenta un análisis bibliométrico de la producción de reseñas editadas en revistas científicas españolas de calidad en ciencias Sociales y Humanidades incluidas en la base de datos ISOC, y publicadas en los años 2007-2009. Se analizan: evolución temporal, revistas editoras, autoría y filiación institucional. También se examina la producción de libros reseñados según tipo documental, idioma de edición, autoría, entidad editora, y la duración del intervalo de tiempo entre edición de libro y reseña. Los resultados sobre reseñas indican un descenso constante en su producción, y un elevado número de documentos en humanidades. Los autores son en su mayoría profesores universitarios y españoles; publican solos; tienen una productividad muy baja; y un alto nivel de endogamia en aquellos autores que son miembros de Consejos de revista. Los resultados sobre libros reseñados demuestran que hay pocos títulos reseñados más de una vez, un elevado número de monografías, muchas en español, y un intervalo alto de tiempo entre la edición de los libros y su reseña.

Palabras clave: Análisis bibliométrico, Ciencias Sociales, Humanidades, reseñas, producción científica, revistas científicas, España, libros.

\section{Book reviews in Spanish scholarly journals in the Social Sciences and Humanities: analysis of production between 2007 and 2009}

Abstract: The goal of this work is to analyse the production of reviews published between
2007 and 2009 in the scholarly journals in the Social Sciences and Humanities contained
in the ISOC database. Different aspects were analysed: time pattern, journals, authorship
and author affiliation. Also examined were the books reviewed, by publication type,
language, authorship, publisher and time lag between the books' publication and the
appearance of the reviews. The results concerning the reviews show a constant reduction
in their production and a large number concentrated in the humanities. Authors are
mostly Spaniards and university lecturers, who publish alone and have a very low level of
productivity. There is also a high level of endogamy among those authors who are members
of editorial boards. The results concerning the reviewed books reveal that few titles were
reviewed more than once, there are a large number of monographs, mostly in Spanish,
and there is a long time lag between a book's publication and the appearance of its review.

Keywords: Bibliometric analysis, Social Sciences, Humanities, reviews, scientific output, scholarly journals, Spain, books.

* Instituto de Estudios Documentales sobre Ciencia y Tecnología (IEDCYT) Centro de Ciencias Humanas y Sociales (CCHS-CSIC), Madrid, España. Correo-e: angela.sorli@cchs.csic.es; gomobez@ yahoo.es; carolinamartincarretero@gmail.com.

Recibido: 04-03-2011; 2. ${ }^{a}$ versión: 14-04.2011; 3. ${ }^{a}$ versión: 29-04-2011; aceptado: 12-05-2011. 


\section{Introducción}

Dentro de los estudios métricos de la información, los artículos de revistas científicas han gozado y gozan de un protagonismo casi absoluto, quedando relegados a un segundo plano los de otros tipos documentales como las monografías y las reseñas bibliográficas. Estas últimas han sido protagonistas de numerosos trabajos, orientados en su mayor parte a servir como guía de una correcta elaboración de las mismas (Lee, 2010; Shaban, 2006; Palmer Bermúdez, 2005) o en forma de estudios sobre las distintas tipologías que pueden presentar (Grant y Booth, 2009), pero se han realizado pocos estudios dedicados a una evaluación cuantitativa de las reseñas, especialmente en España.

Entre los estudios bibliométricos sobre reseñas destacan aquellos realizados sobre las publicadas en revistas de una materia específica durante un período de tiempo muy breve, como es el caso del estudio de Morton (1983) sobre las reseñas publicadas en diversas revistas de medicina durante 1981 y el valor que estas tienen para editores de revistas y de libros; el estudio de Chen, cuyos resultados se publicaron en cinco artículos diferentes (Chen y Wright, 1974 y Chen, 1974a, b, c, d), sobre reseñas de libros de medicina publicadas en 44 revistas de la materia durante 1970; el estudio de Clark y Mai (2000) sobre reseñas publicadas en 1.320 revistas de agricultura y ciencias de la vida durante un año, con el fin servir de elemento de ayuda en la selección de fondos en las bibliotecas. Otros estudios, también realizados en el ámbito anglosajón, han tenido como objetivo un período de tiempo más largo y, en ocasiones, una temática más amplia, como es el caso del estudio de Lindholm-Romantschuk (1998) sobre reseñas realizadas entre 1971 y 1990 a libros publicados por universidades norteamericanas; el análisis de Wheeler Carlo y Natowitz (1995) sobre los criterios de evaluación subjetiva aplicados a las reseñas publicadas en la revista Choice sobre libros de historia americana, geografía y estudios de área entre 1988 y 1993; el estudio de Nicolaisen (2002) sobre reseñas revisadas por pares en seis materias de ciencias sociales durante 30 años; la revisión de reseñas de Murphy y Rehman (1987) sobre la literatura de gestión de empresas en más de 600 títulos de revista entre 1980 y 1983; y el estudio de Nicolaisen y Frandsen (2007) sobre los autores de reseñas en el campo de la economía entre 1985 y 2006 para ejemplificar el potencial efecto del principio del handicap en la investigación en biblioteconomía y documentación.

Otros trabajos de investigación sobre reseñas bibliográficas se han centrado en aspectos puntuales, como las encuestas a autores de reseñas para comprobar la utilidad y las características que estas deberían tener (Hartley, 2005); las expresiones de alabanza en las reseñas publicadas en dos revistas médicas españolas entre 1990 y 2000 con vistas a analizar los contextos y los ejemplos de evaluaciones positivas (Alcaraz Ariza, 2008); la incidencia del sentido positivo o negativo de las críticas en distintos aspectos de las reseñas (Greene y Spornick, 1995) y la evaluación de las reseñas de libros de medicina por bibliotecarios especializados en ciencias de la salud (Martin, 1986). 
Existen diversas fuentes de información en Internet sobre reseñas bibliográficas como son las revistas electrónicas The Bryn Mawr Classical Review, relativas a obras sobre estudios clásicos, y Sehepunkte, sobre obras de historia de la ciencia. También se pueden encontrar bases de datos multidisciplinares sobre reseñas como la Gale Book Review Index Online, y temáticas como la Anthropology Review Database y la Canadian Public Policy Book Reviews Database. En lo que se refiere a España, no existe ninguna base de datos cuya finalidad principal sea recoger información sobre reseñas.

De toda la literatura consultada sobre análisis bibliométrico de reseñas bibliográficas, destaca la ausencia de estudios de amplio espectro dedicados al análisis cuantitativo de este tipo de documentos que hayan sido publicados en revistas científicas españolas o incluso en aquellas del ámbito latinoamericano. Esta ausencia de trabajos y de bases de datos sobre reseñas bibliográficas en español, unido al amplio abanico temático aquí abordado y la base de datos diseñada, le dan al presente estudio un carácter innovador.

El objetivo de este trabajo es analizar distintos indicadores bibliométricos relativos a las reseñas publicadas durante los años 2007, 2008 y 2009 en revistas científicas españolas de Humanidades y Ciencias Sociales de más alta categoría, recogidas en la base de datos ISOC del Centro de Ciencias Humanas y Sociales (CCHS) del CSIC, para comparar los datos y tendencias observados con los que se han obtenido en otros estudios bibliométricos de características similares al que aquí se presenta.

El presente estudio se ha realizado dentro del proyecto de investigación "Establecimiento de indicadores de distinta naturaleza para determinar la calidad de editoriales de monografías científicas en Ciencias Humanas y Sociales", financiado por el CSIC, con el número $200910 I 014$.

\section{Materiales y método}

Para la realización de este estudio se ha acudido a la Base de Datos ISOC de revistas de ciencias sociales y humanidades que mantiene el Centro de Ciencias Humanas y Sociales (CCHS), con el objetivo de seleccionar cinco revistas de cada una de las siguientes áreas de Ciencias Sociales y Humanidades: América Latina, Antropología, Arqueología-Prehistoria, Bellas Artes, Biblioteconomía y Documentación, Ciencias de la Educación, Ciencias Jurídicas, Ciencias Políticas, Economía, Filosofía, Geografía, Historia, Lingüística, Literatura, Miscelánea, Psicología, Sociología y Urbanismo. De las revistas presentes en esa base de datos se han seleccionado aquellas que, a fecha 1 de marzo de 2010, cumpliesen las cotas más altas de calidad (niveles A y B) según los criterios de categorización aplicados a la base de datos ISOC, que han sido elaborados por el Grupo de Investigación de Evaluación de Publicaciones Científicas (EPUC) del IEDCYT (CSIC). Las revistas de algunas materias no alcanzan los requisitos requeridos, por lo que la cifra de cinco publicaciones deseada se ha tenido que restringir a cuatro en Antropo- 
logía, a tres en Ciencias Políticas, y a dos en Psicología y Urbanismo. Debido a esta circunstancia sólo se han podido reunir un total de 81 revistas científicas españolas de Ciencias Sociales y Humanidades de alta calidad que publiquen reseñas bibliográficas (véase listado en Anexo I): Este número supone un 30,22\% del total de revistas de la base de datos ISOC. El período cronológico considerado se ha limitado a los tres años anteriores al inicio de la investigación, esto es, a 2007, 2008 y 2009.

Tras fijar la lista de revistas fuente, se procedió a la inclusión de las referencias de las reseñas bibliográficas y de los libros revisados en una base de datos desarrollada ad hoc que contiene las siguientes tablas principales:

- Tabla Reseña. Recoge los datos sobre las reseñas analizadas. Incluye los siguientes campos:

- Título Original: Es el título de la reseña, en el caso de que lo hubiera.

- Autor: Recoge el nombre y apellidos de cada uno de los autores del documento.

- Lugar de trabajo: Recoge el lugar de trabajo de cada uno de los autores.

- Título Sitio: Se trata del título de la revista en la que aparece publicada la reseña.

- Año de publicación de la revista.

- Volumen de la revista.

- Fascículo de la revista.

- Páginas: página de inicio y fin de la reseña.

- ISSN de la revista

- Cod. ISOC: recoge el código numérico asignado a cada revista en la base de datos ISOC.

- Editorial Revista: se trata de la institución que edita la revista.

- Url: recoge la dirección de la página web en la que se encuentra el texto de la reseña, en caso de que la revista tuviera versión electrónica.

- Tabla Libro. Recoge los datos sobre los libros reseñados tal y como se muestran en sus referencias en las reseñas. Incluye los siguientes campos:

- ISBN del libro reseñado.

- Título Español: contiene el título del libro traducido al español, en el caso de que esté escrito en otra lengua.

- Título Original: contiene el título del documento en su lengua original.

- Autores: nombre y apellidos de cada uno de los autores.

- Editoriales: recoge la entidad o entidades responsables de la edición.

- Año: se refiere al año de su publicación.

- Lengua de publicación.

- Tipología: es su tipo de documento.

- Materias: define su materia.

- Notas. 
Una vez incluidos los datos, se procedió a normalizar los nombres de autores, de las editoriales de libros y de las instituciones, tanto en lugares de trabajo de los autores como en las editoras de revistas, con el fin de que los recuentos obtenidos en cada uno de los campos de la base de datos fueran lo más fieles posibles a la realidad.

De la información introducida en la base de datos se han identificado diferentes indicadores de producción relativos tanto a reseñas como a libros. En lo que se refiere a las reseñas, se han observado los siguientes indicadores: evolución temporal de la producción; productividad en revistas por anualidades, por tipología, por disciplinas y por distribución geográfica de entidades editoras; productividad de autores, índice de coautoría y presencia en Consejos de revistas; productividad institucional, expresada por tipos de institución y por áreas geográficas. Sobre la información de los libros recogidos se ha considerado el número de títulos y la repetición de sus reseñas, la tipología documental, autoría e idiomas de publicación, la tipología y distribución geográfica de las entidades editoras, y el intervalo temporal entre publicación del libro y su reseña.

\section{Resultados}

\subsection{Reseñas}

A través de las ochenta y una revistas fuente estudiadas se han recogido 3.613 reseñas elaboradas por 2.230 autores, que han sido publicadas durante el período comprendido entre 2007 y 2009. De su análisis se han obtenido los siguientes resultados:

\section{Evolución temporal de la producción}

La producción de reseñas por anualidades muestra una leve tendencia al descenso durante el período analizado: en 2007, 1.273 (35,23\%); en 2008, 1.200 $(33,21 \%)$; y en 2009, $1.140(31,55 \%)$.

Si se toman las cifras anuales de producción y se dividen por materias no se observa el leve descenso señalado, si no que se distinguen patrones diferentes dependiendo de la disciplina que se trate. Durante el período estudiado hay áreas que aumentan de forma constante su número de reseñas (Ciencias Jurídicas), otras que disminuyen su cantidad también de forma constante (Bellas Artes, Biblioteconomía y Documentación, Ciencias de la Educación, Ciencias Políticas y Filosofía), y otras que muestran altibajos en el número de documentos que recogen (América Latina, Antropología, Arqueología-Prehistoria, Economía, Geografía, Historia, Lingüística. Literatura, Miscelánea, Psicología, Sociología, Urbanismo). 


\section{Productividad en revistas}

La producción de reseñas por título de revista arroja cifras muy dispares. Se observan cantidades muy elevadas, como las correspondientes a la Revista de Literatura y al Anuario de Estudios Medievales, con 168 y 160 reseñas respectivamente, y otras mínimas, como las recogidas en las revistas Documentación de Ciencias de la Información y Arqueología y Territorio Medieval, que solamente publican 2 y 4 documentos durante los tres años considerados. La media de reseñas por revista obtenida es de 44,6, aunque 50 de las 81 publicaciones revisadas no llegan a alcanzar esa cifra. Esto es debido a la concentración de 1.835 reseñas (el 50,79\% del total) publicadas en un grupo de 20 revistas $(20,25 \%)$.

La distribución de documentos por materias pone de manifiesto que las revistas de Humanidades recogen un mayor número de documentos que aquellas que quedan dentro del ámbito de las Ciencias Sociales (Figura 1). Por un lado, en el área de Humanidades se encuentran materias con un alto número de reseñas, como son la Historia con 476 (13,17\%), la Literatura con 403 (11,15\%) y la Filosofía con $313(8,66 \%)$; mientras que por la otra, se presentan disciplinas de Ciencias Sociales con una exigua producción documental, como son los casos de la Psicología con solamente 38 documentos (1,05\%), la Biblioteconomía y Documentación con 55 (1,52\%), la Antropología con 57 (1,57\%) y el Urbanismo con $60(1,66 \%)$. En el caso de estas dos últimas materias se podría pensar que su número de reseñas es reducido al haberse recogido solamente dos títulos de revista en cada una de ellas, pero la media de reseñas por publicación para cada una de estas materias (28,5 para la Antropología y 30 para el Urbanismo) es bastante baja si la comparamos con la media general por revista señalada más arriba.

La diferencia de producción documental por materias se puede apreciar también al tomar las diez revistas que más reseñas han publicado, de las cuales siete pertenecen a disciplinas de Humanidades, concretamente a Literatura, Historia y Filosofía, y otras, incluidas en las Ciencias Sociales, como Ciencias de la Educación o Ciencias Jurídicas (véase figura 1).

La tipología de las entidades editoras de revistas, todas ellas españolas, abarca un amplio espectro de instituciones, pero con una gran concentración en las categorías Universidades e Institutos de investigación. El grupo más importante está formado por 34 revistas editadas por diversas universidades españolas, entre las que destaca la Universidad Complutense de Madrid con 13 títulos, al que le sigue en orden de importancia un grupo formado por 19 revistas editadas por distintos institutos del CSIC, publicaciones que en su mayor parte pertenecen al área de Humanidades. El segundo grupo más numeroso recoge 10 revistas editadas por organismos de la Administración Pública, que se incluyen dentro del área de Ciencias Sociales, concretamente en Economía, Ciencias Jurídicas y Ciencias Políticas, y un grupo de 7 revistas editadas por asociaciones y colegios profesionales, también con una mayoría de publicaciones del área de Ciencias Sociales. Por último, se encuentran las revistas editadas por editoriales comerciales (4), 
FIGURA 1

\section{Producción de reseñas por materias}

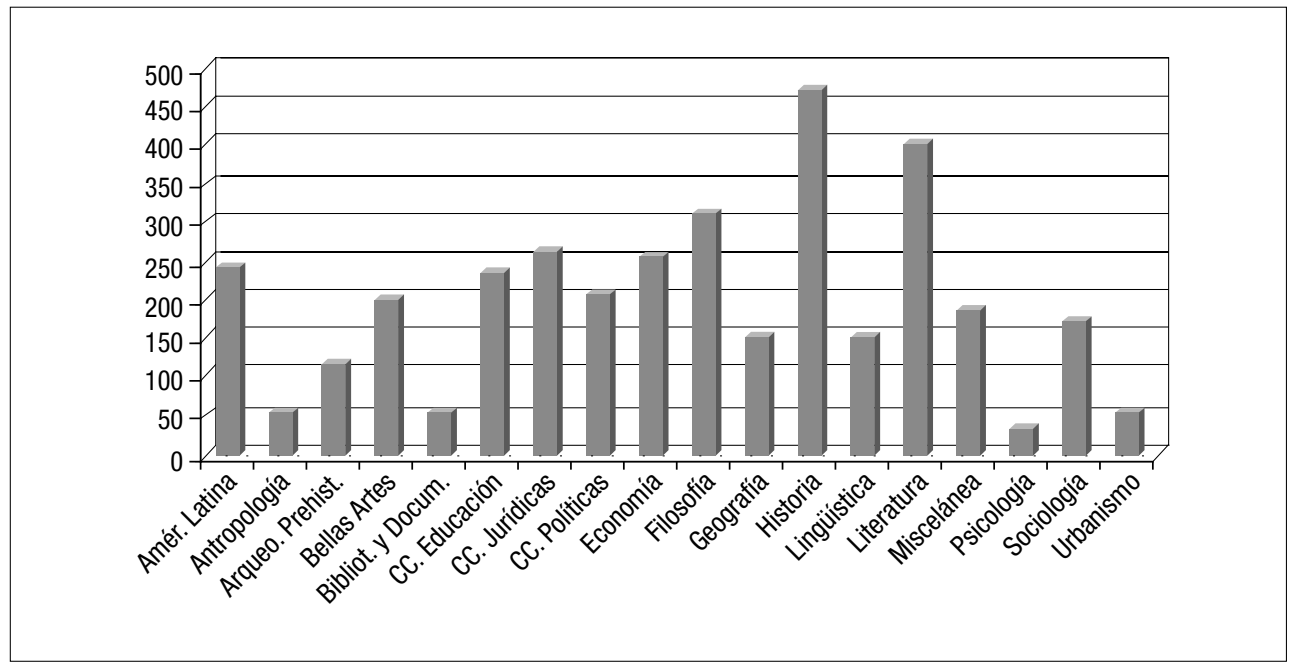

fundaciones (3) y otras entidades (4), que recoge un centro educativo, una Real Academia y un instituto sobre educación.

A pesar de que las revistas editadas por universidades son mayoría $(41,98 \%)$ en el conjunto de publicaciones sometidas a examen, son las revistas editadas por los institutos de investigación del CSIC las que publican un mayor número de reseñas, tal y como se aprecia en la figura 2. Estas revistas son responsables de la edición de 1.413 reseñas frente a las 1.036 que se han editado en aquellas publicaciones realizadas en las universidades. El resto de las categorías de entidades editoras reúnen un número bastante menor de documentos publicados: Colegios y Asociaciones Profesionales (235), Editoriales (76), Fundaciones (115), Organismos de la Administración Pública (621) y Otros (117).

\section{Productividad de autores}

De los 2.230 autores de reseñas que se han identificado, un 96,95\% publican de forma individual, mientras que un 3,05\% lo hacen como coautores de trabajos de reseña. La coautoría solamente se da en 33 reseñas, de las cuales treinta y dos han sido elaboradas por dos autores y una, por cuatro. De todos los trabajos en colaboración, un 63,89\% pertenecen a revistas de materias del ámbito de las Ciencias Sociales y un 36,11\% a títulos de Humanidades.

En cuanto a la productividad por autor, señalar que 1.639 han publicado un único trabajo, lo que deja una cantidad muy reducida de autores (591) que son responsables de más de una reseña. De estos últimos, un 60,41\% son responsables de dos reseñas, un 19,29\% de tres, y un 20,3\% de cuatro o más. El grupo 


\section{FIGURA 2}

\section{Porcentajes de producción de reseñas por tipo de institución editora de revista}

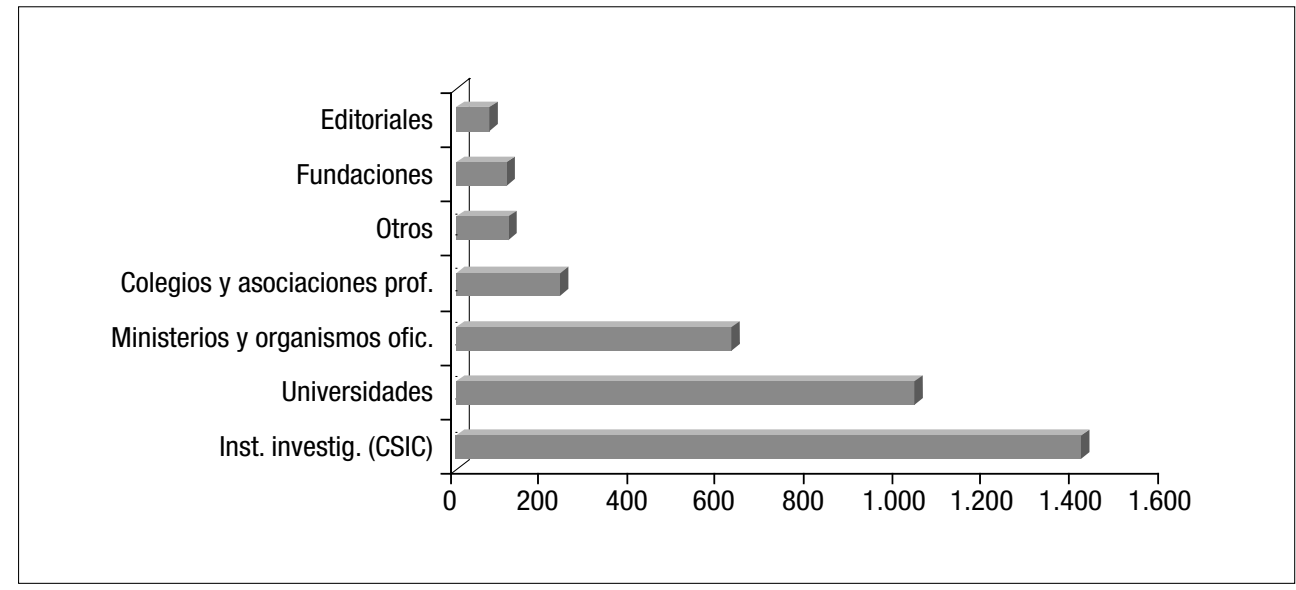

más prolífico está formado por 120 autores a los que se deben 982 reseñas, un $27,18 \%$ del total de trabajos, y entre los que se encuentran cinco autores que han reseñado cantidades que van desde los 24 hasta los 72 libros. Esta circunstancia hace que la media de trabajos por autor aumente, aunque no de manera significativa.

Como consecuencia del alto porcentaje de reseñadores que no repiten autoría, el número medio de obras por autor resulta una cifra bastante escasa: 1,61. Si se atiende a cada una de las materias por separado, se aprecia que hay tres materias con una media muy baja: Biblioteconomía y Documentación $(1,10)$, Psicología $(1,11)$ y Sociología $(1,16)$. Por contra, la media de estas cuatro materias es bastante más elevada que la general: Bellas Artes $(1,86)$, Historia $(1,89)$, Economía $(2,87)$ y Urbanismo $(7,50)$. En esta última disciplina la media es muy superior a la general, pero ello es debido a que la practica totalidad de las reseñas están escritas por la misma autora.También se ha advertido que entre el personal de los Consejos de Redacción y asesores de casi todas las revistas estudiadas se encuentran 268 autores de reseñas, un 12,02\% del total analizado, que son responsables del 23,28\% del total de las revisiones bibliográficas consideradas. De estos 268 autores, 241 (89,92\%) escriben exclusivamente para aquellas revistas de cuyos Consejos forman parte.

\section{Productividad institucional}

El estudio de la productividad institucional presenta un problema, y es que en el 49,21\% de los trabajos no se recoge el organismo de trabajo del autor. Esta ausencia de filiación institucional se da en todos los documentos de 20 revistas 
y de forma parcial en los contenidos en otras 46. Solamente en 15 publicaciones se especifica la institución de trabajo de todos sus autores. La obtención de datos sobre la distribución geográfica de instituciones y su tipología se ha hecho pese a esta falta de información existente en casi la mitad de los documentos.

En la producción por tipo de institución, como se puede apreciar en la figura 3, hay una mayor presencia de los escritos debidos al personal de las universidades $(74,22 \%)$ frente a la más reducida que aportan los miembros de los institutos de investigación científica $(21,09 \%)$, que en su práctica totalidad pertenecen al CSIC (369 de 387 reseñas), y al escaso número de autores de instituciones de la Administración Pública (2,23\%) y otras entidades (2,45\%), entre las que se encuentran museos, centros educativos, bibliotecas, asociaciones culturales y empresas privadas.

\section{FIGURA 3}

\section{Tipos de instituciones de trabajo de los autores de reseñas}

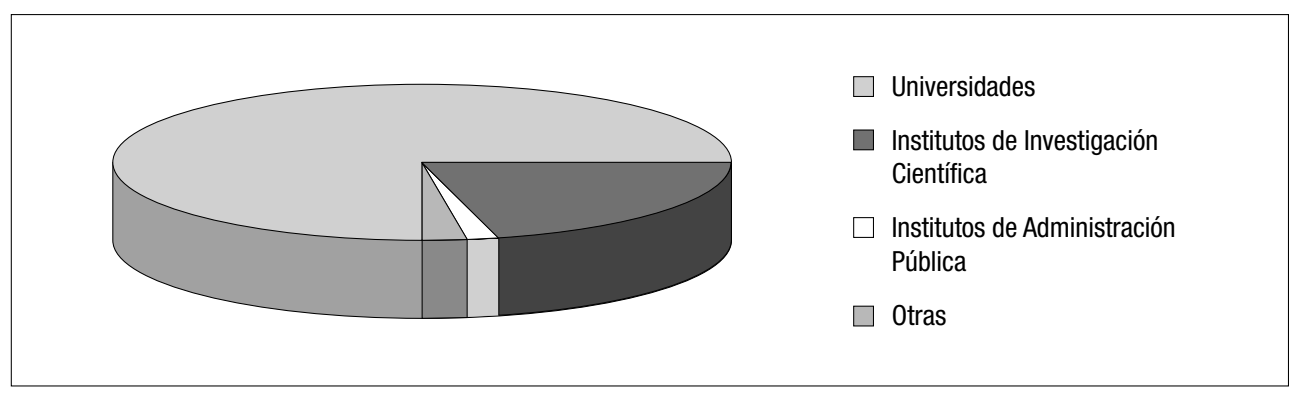

En lo que a la distribución geográfica de instituciones se refiere, y dado que la única lengua utilizada en la redacción de las reseñas es el castellano, destaca España como el país de procedencia de un número muy elevado de documentos: 1.647. Le siguen, a considerable distancia, los Estados Unidos con 38 reseñas, el Reino Unido con 20, Argentina con 18, Francia con 16, Italia con 15, México con 14 y Perú con 11.

Si se toman las 1.647 reseñas elaboradas por personal de instituciones españolas, se constata que la mayoría de los autores trabajan en instituciones radicadas en la Comunidad de Madrid (51,9\%). Dichas instituciones también son mayoría en cada uno de los tipos de institución que se han especificado más arriba: 513 en Universidades, 291 en Institutos de investigación, 32 en Organismos de la Administración Pública y 19 en otros. Entre las restantes comunidades autónomas con mayores porcentajes de documentos están Andalucía (12,08\%), con 181 reseñas elaboradas por personal adscrito en su mayor parte a las universidades; Cataluña (10,20\%), donde destacan las 67 reseñas debidas al personal de institutos del CSIC; Castilla y León (6,25\%); y la Comunidad Valenciana $(4,22 \%)$. 


\subsection{Libros reseñados}

En este apartado se recogen distintos aspectos relativos a los libros reseñados en las ochenta y una revistas estudiadas, entendiendo por "libro", a modo de convención, cada unidad documental que se pueda incluir en los siguientes seis tipos: monografías, actas de congresos, tesis doctorales, informes, normas y homenajes. Los datos que se presentan han sido extraídos de las referencias bibliográficas de la obra que acompaña a las reseñas.

\section{Producción por libros}

Se han recogido 3.372 títulos de libros, de los cuales solamente un 6,37\% han sido reseñados más de una vez, quedando distribuidas las cantidades de estos últimos de la siguiente manera: 189 (5,60\%) han sido reseñados dos veces, 23 $(0,68 \%)$ lo han sido tres veces y $3(0,09 \%)$ cuatro veces.

Las cantidades de cada tipología documental que se han establecido para las obras reseñadas son muy escasas, excepto en el caso de las monografías. Se han contabilizado 3.266 monografías, 72 actas de congresos, 18 homenajes, 2 informes, 2 normas y 12 sin tipo documental.

En la distribución de documentos por idioma se observa que es el español la lengua con un porcentaje más alto (76,37\%), dejando en unos niveles muy bajos a otros idiomas de publicación. Los porcentajes de otras lenguas relevantes son los siguientes: inglés $(9,80 \%)$, francés $(4,08 \%)$, italiano (4\%), catalán $(2,27 \%)$, alemán $(1,55 \%)$ y otros $(1,84 \%)$. Si se consideran los libros por la lengua de publicación y la materia que tratan, se puede comprobar que los únicos idiomas que están presentes en todas las disciplinas son el español y el inglés.

Si se eliminan aquellas obras sin autoría conocida y las duplicadas de entre los trabajos reseñados, se recogen 4.037 nombres de autores sobre un total de 3.293 libros. Entre todas estas obras se han hallado 2.458 realizadas por un único autor, 604 por dos autores, 163 por tres, 43 por cuatro, 25 por cinco o más autores.

\section{Producción por entidades editoras}

Con el fin de comprobar la producción de los diferentes sectores editoriales, se han agrupado las 1.525 entidades editoras contabilizadas en seis tipos distintos, que a continuación se relacionan por orden de porcentaje: editoriales comerciales $(51,10 \%)$, universidades (23,33\%), organismos de la administración pública (8,84\%), fundaciones e instituciones de estudios (6,82\%), academias e institutos de investigación $(5,93 \%)$ y otros $(3,98 \%)$, entre los que se encuentran museos, asociaciones y colegios profesionales, unidades de información, entidades bancarias, colegios e institutos de enseñanza.

En lo que se refiere a la distribución geográfica de las entidades editoras destacan las españolas con un porcentaje del 57,24\%. Con una menor cantidad están los porcentajes de las editoriales del conjunto de países latinoamericanos 
$(12,85 \%)$, de las editoriales italianas $(6,62 \%)$, francesas $(5,31 \%)$, norteamericanas (4,85\%), alemanas (3,01\%) y británicas (2,95\%). Si se consideran las entidades editoras con mayor número de libros reseñados se puede comprobar que la preponderancia de las editoriales españolas, quedando las extranjeras en un nivel muy bajo, como se puede observar en la figura 4.

\section{FIGURA 4}

\section{Entidades editoras con mayor número de libros reseñados}

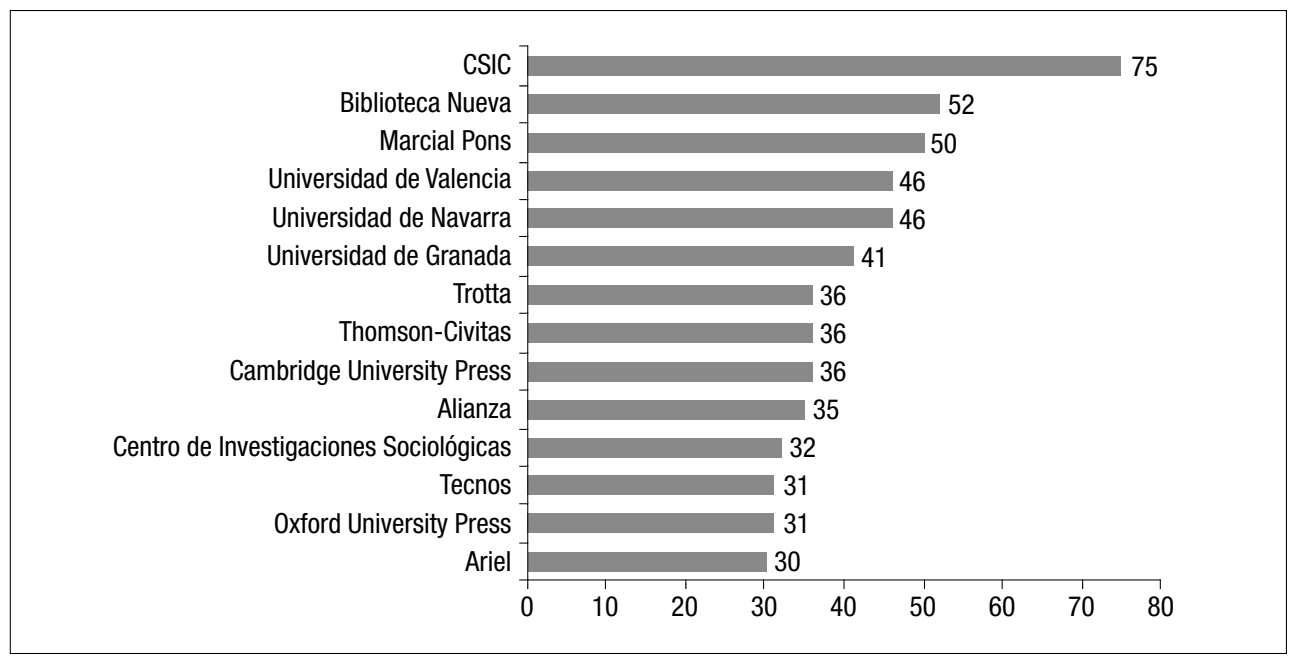

Los porcentajes anuales de edición de libros tienen sus mayores valores en los años 2006 (26,15\%), 2007 (28,84\%) y 2008 (18,98\%), mientras que los correspondientes a las anualidades anteriores y posteriores a este período quedan en niveles mucho menores. En 2004 y 2005 los porcentajes caen hasta un 4,75\% y un 11,86\%, respectivamente, por la antigüedad de los libros, y en 2009 queda en 4,34\% por el grado de actualidad de lo publicado, ya que este es el último año del estudio. También se observan datos sobre libros publicados en el siglo xx, e incluso el xIx, los cuales pertenecen en su mayoría a Historia, Geografía y Filosofía, que son las materias con mayor intervalo medio de tiempo transcurrido entre la edición de los libros y la publicación de sus reseñas (véase figura 5).

El cálculo del intervalo de tiempo transcurrido entre la edición de un libro y la publicación de su reseña se realiza solamente en años, porque ese es el único dato que se conoce de la fecha de edición de los libros aquí tratados. La media general resultante para este intervalo de tiempo es de 1,63 años. Ahora bien, si se observan los resultados agrupados por materias en la figura 6 , se puede comprobar que existe una gran disparidad pues Historia $(2,51)$, Filosofía $(2,17)$ y Geografía $(2,11)$ hay intervalos muy largos, mientras que en Urbanismo $(0,81)$ y Biblioteconomía y Documentación $(0,89)$ no superan el año entre edición y reseña. 
FIGURA 5

Número de libros reseñados por año de edición

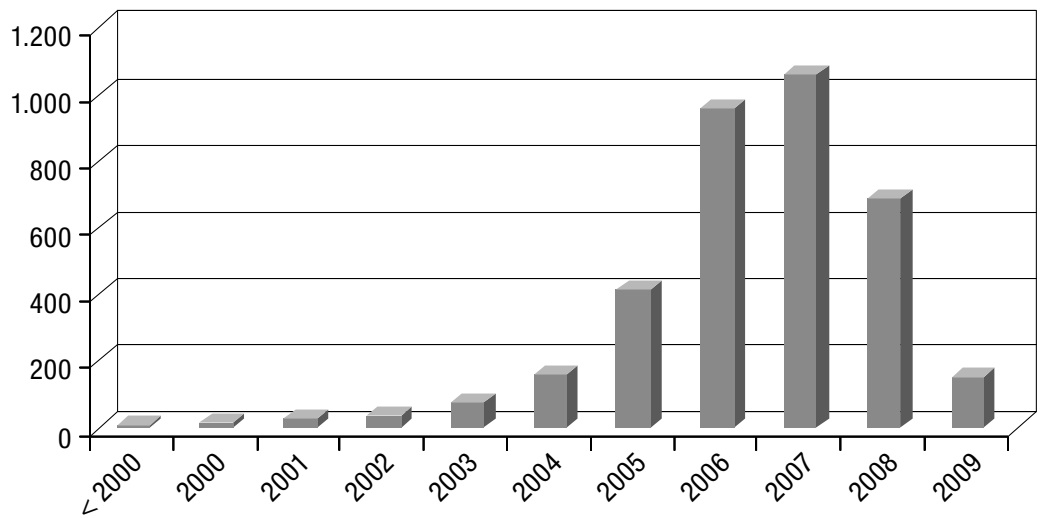

FIGURA 6

Intervalo de tiempo entre año de edición de libro y su reseña por materias

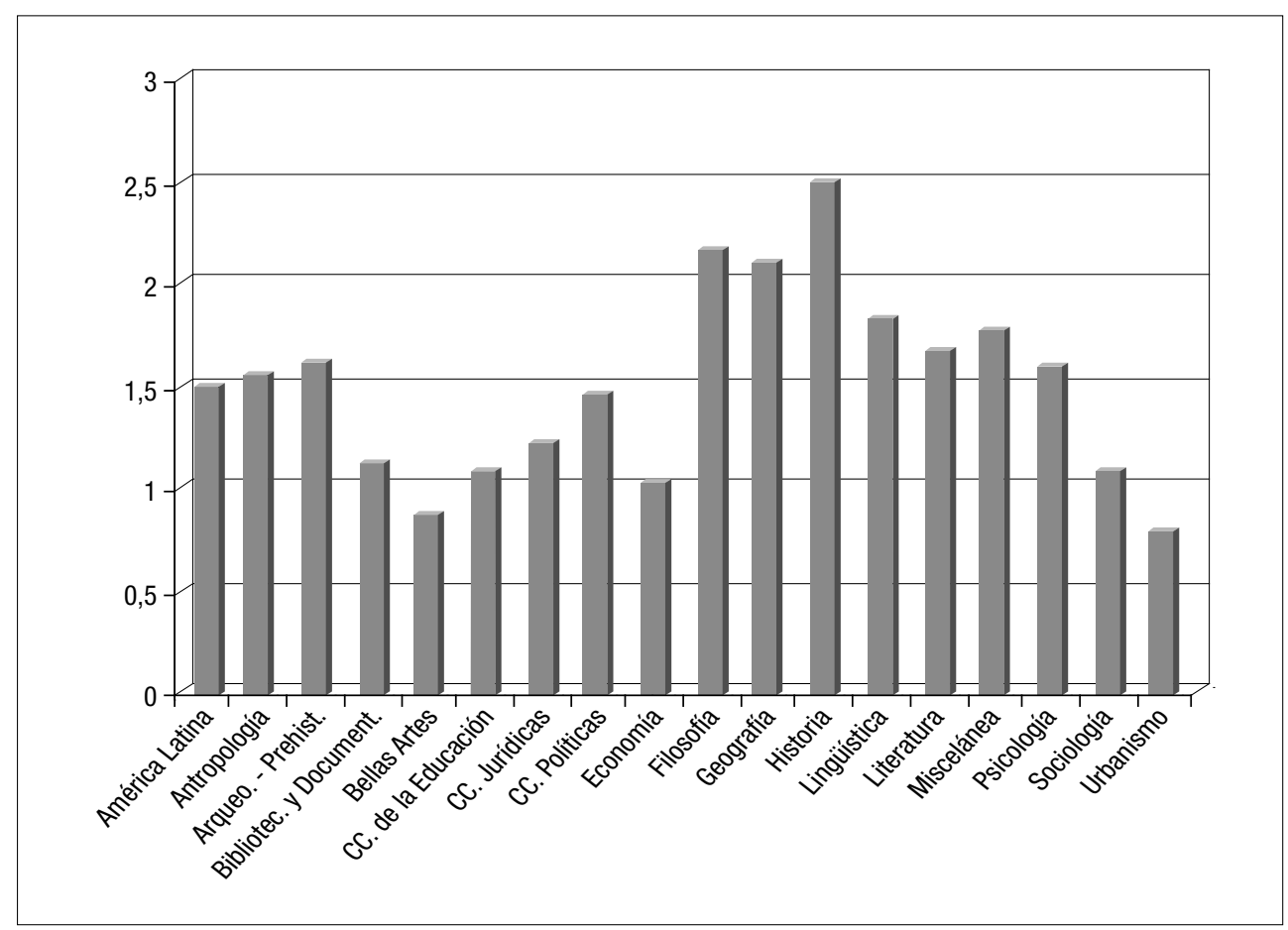




\section{Discusión y conclusiones}

Hasta el momento actual no se había acometido ningún estudio que tuviese unos objetivos similares a los del presente trabajo, esto es, el análisis bibliométrico multidisciplinar de reseñas en lengua española presentes en revistas científicas. Las reseñas han sido objeto de diversos estudios bibliométricos durante las últimas décadas, siendo realizados en su inmensa mayoría en el ámbito anglosajón, y a menudo circunscritos a revistas de una disciplina o centrados en una única publicación periódica. Es por ello por lo que ha resultado complicado establecer comparaciones entre los resultados aquí obtenidos y los procedentes de otras investigaciones. A pesar de la diferencia temática y del número de publicaciones que tratan, cuando ha sido posible se han recogido resultados procedentes de diferentes investigaciones consultadas para comprobar las afinidades y diferencias que estas pueden tener con los datos observados por los autores.

Las reseñas estudiadas presentan una productividad a la baja que puede resultar desigual según los parámetros de análisis que se apliquen. Si se atiende a la evolución temporal de la producción, se puede comprobar una paulatina disminución del 5\% anual $(5,73 \%$ de descenso en 2008 respecto de la cantidad de 2007 y 5\% en 2009 respecto de la cantidad de 2008) en la producción de reseñas en revistas científicas españolas. Este descenso puede interpretarse como falta de interés por este tipo de documento, detalle ya señalado por diversos autores (Lindholm-Romantschuk, 1998; Sabosik, 1988; Diodato, 1988).

En el caso de la producción de documentos por revista se encuentran cantidades muy bajas en unas, mientras que en otras se dan grandes acumulaciones. Entre estas últimas se encuentra un núcleo de 20 revistas (20,25\%) en el que se recogen la mitad del total de reseñas consideradas. Dicho núcleo tiene una cantidad elevada de documentos aunque no llega nivel de otros estudios como el de Murphy y Rehman (1987) sobre libros de gestión empresarial, en el que observan un pequeño grupo de revistas (10\% del total) en las que se publican un $53 \%$ de las reseñas del estudio; el estudio sobre revistas de Medicina de Chen y Wright (1970), en el que un conjunto de revistas (13\% de las observadas) contiene el 52,8\% de los documentos estudiados; o la obra de Lindholm-Romantschuk (1998), en la que se sostiene que en menos del 20\% de las revistas sobre distintas disciplinas se alcanzan el $76 \%$ del total de reseñas evaluadas. Al tomar las revistas por disciplinas, el porcentaje de documentos pertenecientes a las materias de Humanidades resulta el más elevado, y de forma particular, la Historia. Este último dato coincide con lo observado por Lindholm-Romantschuk (1998) en su estudio de reseñas de libros publicados por universidades norteamericanas.

Si se consideran las revistas atendiendo al tipo de institución editora, resultan ser los institutos de investigación, concretamente aquellos que pertenecen al CSIC, los que publican mayor número de reseñas. La productividad de estos institutos es superior a la alcanzada por las universidades españolas, y ello a pesar de que el número de títulos de revista editados por los institutos del CSIC es muy inferior al de estas. 
Dentro de los parámetros referidos a la autoría destaca la inmensa cantidad de documentos con un único autor, conclusión a la que también llega Alcaraz Ariza (2008) en su estudio sobre reseñas de Medicina. Se constata que la producción de reseñas en las áreas de conocimiento analizadas es más una labor individual que el fruto del trabajo en colaboración, especialmente en aquellas disciplinas de Humanidades. Junto a este dato sobre el bajo índice de coautoría, se aprecia también un escaso número de autores responsables de más de un documento, lo que provoca una exigua media de reseñas por autor: 1,61. También entre los autores se observa preferencia por las revistas a las que están vinculados a la hora de publicar. El nivel de endogamia al que se llega es enorme cuando se comprueba que la mayor parte de autores vinculados al Consejo de alguna revista sólo escriben para dicha publicación.

Respecto a la producción institucional se concluye que la mayor parte corresponde al personal de las universidades frente al resto de instituciones en una relación de 3 a 1. Entre el resto de instituciones se encuentran los institutos del CSIC, cuyo personal tiene una producción más baja de lo que se puede esperar viendo los medios de publicación de los que disponen. Porque no hay que olvidar, como ya se ha señalado antes, que las revistas del CSIC son las que más reseñas publican.

En relación a la ubicación de las instituciones de los autores y dado que el único idioma de las reseñas es el español, no sorprende que la mayoría de las entidades sean españolas, pero sí llama la atención el gran número de instituciones radicadas en la Comunidad de Madrid y el escaso porcentaje de trabajos elaborados por personal de instituciones latinoamericanas.

Los datos sobre los libros reseñados muestran un perfil de autor que publica monografías de forma no cooperativa, y preferentemente en lengua española. La monografía es, con mucho, la forma documental más reseñada, como señala Alcaraz Ariza (2008), quizá por el interés que despierta entre los autores de Ciencias Sociales y Humanidades como vehículo de transmisión de los resultados de sus investigaciones. Los autores de estas áreas del conocimiento también prefieren de forma mayoritaria, especialmente los de las disciplinas de Humanidades, publicar en solitario frente a la obra en colaboración. Esto se puede apreciar en el alto porcentaje de libros de un único autor $(74,64 \%)$, lo que da lugar a una media de obras por autor realmente baja: 1,22. Por último, el idioma de edición más común observado es el español, quedando el inglés en un número muy bajo de documentos. Lo que puede ser interpretado como un afán localista frente a las novedades editoriales.

Los títulos de libros que han recibido más de una reseña representan un mínimo porcentaje $(6,37 \%)$ respecto del total de documentos reseñados, que se puede considerar mínimo si se compara con los 19,41\% y 35,17\% obtenidos en los estudios de Morton (1983) y Chen (1974b). La existencia de una única opinión sobre cada novedad literaria limita al lector a la hora de formarse un juicio sobre la calidad de la obra.

La observación de parámetros relativos a las entidades editoras de los libros ha permitido conocer detalles sobre su tipología, procedencia geográfica y el 
grado de novedad de las obras revisadas. Respecto al primer parámetro, se constata la existencia de una mayoría de editoriales comerciales y un número relativamente importante de editoriales de universidades, superior en porcentaje al obtenido en otro estudio (Chen, 1974c). En cuanto a la procedencia geográfica, la gran cantidad de entidades de España y el bajo número de editoriales de países europeos y Estados Unidos dan la impresión de que los libros a reseñar se restringen al ámbito local, como se apuntaba más arriba.

Otro parámetro observado, y quizá el más importante pues da una medida del valor de la revista reseñadora (Chen, 1974a), es el intervalo de tiempo transcurrido entre la publicación del libro y su reseña. La media obtenida en este caso, 1,63 años, es muy superior a las obtenidas en estudios sobre libros de ciencias de la salud (Chen 1974a y Morton, 1983), pero son similares a las publicadas en trabajos sobre obras de carácter multidisciplinar (Clark y Mai, 2000 y Lindholm-Romantschuk, 1998). Por otra parte, conviene señalar lo chocante que resulta la presencia de reseñas realizadas a libros editados hace décadas, dado que uno de los requisitos recogidos en las normas a autores de las revistas sobre las reseñas a publicar es que el libro sometido a revisión sea una novedad editorial.

\section{Bibliografia}

Alcaraz-Ariza, M. A. (2008). Las reseñas de libros médicos escritos en español: un estudio sociopragmático de las expresiones de alabanza. Ibérica, 16, 37-58.

Clark, K. A., y Mai, B. A. (2000). Locating book reviews in agriculture and the life sciences. Science \& Technology Libraries, vol. 18 (4), 3-27.

Chen, C. C., y Wright, A. M. (1974). Current status of biomedical book reviewing: Part I. Key biomedical reviewing journals with quantitative significance. Bulletin of the Medical Library Association, 62 (2), p. 105-112.

Chen, C. C. (1974a). Current status of biomedical book reviewing: Part II. Time lag in biomedical book reviewing. Bulletin of the Medical Library Association, 62 (2), 113-119.

Chen, C. C. (1974b). Current status of biomedical book reviewing: Part III. Duplication patterns in biomedical book reviewing. Bulletin of the Medical Library Association, 62 (3), p. 296-301.

Chen, C. C. (1974c). Current status of biomedical book reviewing: Part IV. Major American and British book publishers. Bulletin of the Medical Library Association, 62 (3), 302-308.

Chen, C. C. (1974d). Current status of biomedical book reviewing: Part V. Most frequently reviewed biomedical books in 1970. Bulletin of the Medical Library Association, 62 (3), 309-313.

Diodato, V. (1988). Impact and scholarliness in arts and humanities book reviews: a citation analysis. Challenges to an information society. Proceedings of the $47^{\text {th }}$ ASIS annual meeting, p. 217-220. White Plains, NY: Knowledge Industry Publications.

Grant, M. J., y Booth, A. (2009). A typology of reviews: an analysis of 14 review types and associated methodologies. Health Information and Libraries Journal, 26, 91-108.

540 Rev. Esp. Doc. Cient., 34, 4, octubre-diciembre, 526-544, 2011. ISSN: 0210-0614. doi: 10.3989/redc.2011.4.841 
Greene, R. J., y Spornick, C. D. (1995). Favorable and unfavorable book reviews: A quantitative study. The Journal of Academic Librarianship, Vol. 21, n. ${ }^{\circ}$ 6, 449-453.

Hartley, J. (2005). Book reviewing in the BJET: a survey of BJET's referees' and writers' views. British Journal Journal of Educational Technology, vol. 36 n. ${ }^{\circ}$ 5, 897-905.

Lee, A. D.; Green, B. N.; Johnson, C. D., y Nyquist, J. (2010). How to write a scholarly book review for publication in a peer-reviewed journal: a review of the literature. The Journal of Chiropractic Education, vol. 24, n. ${ }^{\circ}$ 1, 57-69.

Lindholm-Romantschuk, Y. (1998). Scholarly book reviewing in the social sciences and humanities: The flow of ideas within and among disciplines. Wesport: Greenwood Press.

Martin, Lynne, M. (1986). The evaluation of Biomedical Book Reviews by Academic Health Sciences Librarians. Bulletin of the Medical Library Association, 74, 2, 157-160.

Morton, P. Y. (1983). Medical book reviewing. Bulletin of Medical Library Association, vol. 71 (2), 202-206.

Murphy, M., y Renman, S. U. (1987). The reviewing of management literature. Library Quarterly, vol. 57, 32-60.

Nicolaisen, J. (2002) The scholarliness of published peer reviews: a bibliometric study of book reviews in selected social science fields. Research Evaluation, vol. 11, n. ${ }^{\circ}$, 129-140.

Nicolaisen, J., y Frandsen, T. F. (2007). The handicap principle: a new perspective for library and information science research. Information Research, 12 (4). Disponible en http://InformationR.net/ir/12-4/colis/colis23.html [consulta: 21 de enero de 2011].

Palmer Bermúdez, N. (2005). Los elementos fundamentales de una reseña. Puerto Rico: Neyssa Palmer Bermúdez. Disponible en: www.1.uprh.edu/cruzmigu/ESPA_LEFDUR. pdf [consulta: 21 de enero de 2011].

Sabosik, P. A. (1988). Scholarly Publishing and the role of Choice in the post publication review process. Book Research Quarterly, 10-18.

Shaban, R. (2006). A guide to writing book reviews. Journal of Emergency Primary Healt Care, vol. 4, n. $^{\circ} 3,4$.

Wheeler Carlo, P., y Natowitz, A. (1995). Choice book reviews in American history, geography, and area studies: An analysis for 1988-1993. Library Acquisitions: Practice $\&$ Theory, vol. 19, n. ${ }^{\circ} 2,153-165$.

\section{Anexo 1. Revistas objeto de estudio}

\begin{tabular}{l|l}
\hline \multicolumn{1}{c|}{ Título revista } & \multicolumn{1}{c}{ Área temática } \\
\hline Al-Qantara & Miscelánea \\
\hline Anales Cervantinos & Literatura \\
\hline Anales de Literatura Hispanoamericana & América Latina \\
\hline Anales del Seminario de Historia de la Filosofía & Filosofía \\
\hline Anuario de Estudios Americanos & América Latina \\
\hline
\end{tabular}




\begin{tabular}{|c|c|}
\hline Título revista & Área temática \\
\hline Anuario de Estudios Medievales & Historia \\
\hline Anuario de Psicología & Psicología \\
\hline Archivo de Arte Valenciano & Bellas Artes \\
\hline Archivo Español de Arqueología & Arqueología-Prehistoria \\
\hline Archivo Español de Arte & Bellas Artes \\
\hline Arqueología y Territorio Medieval & Arqueología-Prehistoria \\
\hline Artigrama & Bellas Artes \\
\hline Awraq & Miscelánea \\
\hline Boletín de la Asociación de Geógrafos Españoles & Geografía \\
\hline Ciudad y Territorio, Estudios Territoriales & Urbanismo \\
\hline $\begin{array}{l}\text { Ciudades. Revista del Instituto de Urbanistica de la Univer- } \\
\text { sidad de Valladolid }\end{array}$ & Urbanismo \\
\hline Complutum & Arqueología-Prehistoria \\
\hline Crónica Tributaria & Economía \\
\hline Cuadernos de Arte de la Universidad de Granada & Bellas Artes \\
\hline $\begin{array}{l}\text { Cuadernos de Filología Clásica. Estudios Griegos e Indo- } \\
\text { europeos }\end{array}$ & Literatura \\
\hline Cuadernos de Filología Clásica. Estudios Latinos & Literatura \\
\hline Cuadernos de Historia Moderna & Historia \\
\hline Cuadernos Geográficos & Geografía \\
\hline Daimon. Revista de Filosofía & Filosofía \\
\hline Derechos y Libertades & Derecho \\
\hline Documentación de las Ciencias de la Información & Biblioteconomía y Documentación \\
\hline Dynamis & Historia \\
\hline Economistas & Economía \\
\hline El Profesional de la Información & Biblioteconomía y Documentación \\
\hline Emerita. Revista de Lingüística y Filología Clásica & Literatura \\
\hline Ería & Geografía \\
\hline Estudios de Psicología & Psicología \\
\hline $\begin{array}{l}\text { Estudios financieros. Revista de contabilidad y tributación. } \\
\text { Comentarios, casos prácticos }\end{array}$ & Economía \\
\hline Estudios Geográficos & Geografía \\
\hline
\end{tabular}


Reseñas en revistas científicas españolas de Ciencias Sociales y Humanidades: análisis...

\begin{tabular}{|c|c|}
\hline Título revista & Área temática \\
\hline Goya & Bellas Artes \\
\hline Habis & Miscelánea \\
\hline Hispania & Historia \\
\hline Hispania Sacra & Historia \\
\hline Iber. Didáctica de las Ciencias Sociales, Geografía e Historia & Ciencias de la Educación \\
\hline Información Comercial Española. Revista de Economía & Economía \\
\hline Isegoría & Filosofía \\
\hline Item. Revista de Biblioteconomía i documentació & Biblioteconomía y Documentación \\
\hline Ius Canonicum & Derecho \\
\hline Lenguaje y Textos & Lingüística \\
\hline Minerva. Revista de Filología Clásica & Lingüística \\
\hline $\begin{array}{l}\text { Miscelánea de Estudios Árabes y Hebraicos. Sección de He- } \\
\quad \text { breo }\end{array}$ & Miscelánea \\
\hline Papeles de Geografia & Geografía \\
\hline Papers. Revista de Sociología & Sociología \\
\hline Pedagogía Social. Revista interuniversitaria & Ciencias de la Educación \\
\hline $\begin{array}{l}\text { Pensamiento. Revista de Investigación e Información Filo- } \\
\quad \text { sófica }\end{array}$ & Filosofía \\
\hline Politica y Sociedad & Sociología \\
\hline Psicothema & Psicología \\
\hline RES. Revista Española de Sociología & Sociología \\
\hline Revista Complutense de Historia de América & América Latina \\
\hline Revista de Administración Pública & Derecho \\
\hline Revista de Antropología Social & Antropología \\
\hline Revista de Dialectología y Tradiciones populares & Antropología \\
\hline Revista de Economía Industrial & Economía \\
\hline Revista de Educación & Ciencias de la Educación \\
\hline Revista de Estudios Políticos & Ciencias Políticas \\
\hline Revista de Filología Española & Lingüística \\
\hline Revista de Indias & América Latina \\
\hline Revista de Literatura & Literatura \\
\hline Revista Española de Antropología Americana & América Latina \\
\hline
\end{tabular}




\begin{tabular}{l|l}
\hline \multicolumn{1}{c|}{ Título revista } & \multicolumn{1}{c}{ Área temática } \\
\hline Revista Española de Ciencia Política & Ciencias Políticas \\
\hline Revista Española de Derecho Constitucional & Derecho \\
\hline Revista Española de Derecho del Trabajo & Derecho \\
\hline Revista Española de Documentación Científica & Biblioteconomía y Documentación \\
\hline Revista Española de Investigaciones Sociológicas & Ciencias Políticas \\
\hline Revista Española de Lingüística & Lingüística \\
\hline Revista Española de Pedagogía & Ciencias de la Educación \\
\hline Revista General de Información y Documentación & Biblioteconomía y Documentación \\
\hline Revista Internacional de Sociología & Sociología \\
\hline Sefarad & Miscelánea \\
\hline Sistema & Ciencias Políticas \\
\hline Teorema. Revista internacional de filosofía & Filosofía \\
\hline Teoría de la Educación. Revista Interuniversitaria & Ciencias de la Educación \\
\hline Trabajos de Prehistoria & Arqueología-Prehistoria \\
\hline Verba Anuario Galego de Filoloxía & Lingüística \\
\hline Zephyrus & Arqueología-Prehistoria \\
\hline Zer. Revista de Estudios de Comunicación & Sociología \\
\hline &
\end{tabular}

